\title{
OPEN Relationship between initial peritoneal dialysis modality and risk of peritonitis
}

\author{
Maiko Kokubu ${ }^{1}$, Masaru Matsui ${ }^{1 凶}$, Takayuki Uemura ${ }^{1}$, Katsuhiko Morimoto ${ }^{2}$, \\ Masahiro Eriguchi ${ }^{3}$, Kenichi Samejima ${ }^{3}$, Yasuhiro Akai $^{3}$ \& Kazuhiko Tsuruya ${ }^{3}$
}

Peritonitis is a critical complication of peritoneal dialysis (PD). Investigators have reported the risk of peritonitis in patients on continuous ambulatory peritoneal dialysis (CAPD) versus automated peritoneal dialysis (APD), but the available evidence is predominantly based on observational studies which failed to report on the connection type. Our understanding of the relationship between peritonitis risk and PD modality thus remained insufficient. We studied 285 participants who began PD treatment between 1997 and 2014 at three hospitals in Nara Prefecture in Japan. We matched 106 APD patients with 106 CAPD patients based on their propensity scores. The primary outcome was time to first episode of peritonitis within 3 years after PD commencement. In total, PD peritonitis occurred in 64 patients during the study period. Patients initiated on APD had a lower risk of peritonitis than did those initiated on CAPD in both the unadjusted and adjusted models. The hazard ratio (HR) and $95 \%$ confidence interval $(\mathrm{Cl})$ for the primary endpoint were $0.30(0.17-0.53)$ in the fully adjusted model including connection type. In the matched cohort, APD patients had a significantly lower risk of peritonitis than did CAPD patients (log-rank: $p<0.001, \mathrm{HR} 0.32,95 \% \mathrm{Cl} 0.16-0.59$ ). The weightingadjusted analysis of the inverse probability of treatment yielded a similar result ( $\mathrm{HR} 0.35,95 \% \mathrm{Cl}$ 0.18-0.67). In conclusion, patients initiated on APD at PD commencement had a reduced risk of peritonitis compared with those initiated on CAPD, suggesting APD may be preferable for prevention of peritonitis among PD patients.

Peritonitis is a frequent and serious complication among patients on peritoneal dialysis (PD) and is characterized by fever, severe abdominal pain and cloudy effluents. Some centers report that PD peritonitis accounts for approximately 0.20 episodes per patient-year ${ }^{1,2}$. PD peritonitis is considered a direct or indirect cause of death in $2-16 \%$ of patients $s^{3-5}$. Furthermore, peritonitis greatly reduces dialytic efficiency via peritoneal fibrosis progression $^{6,7}$ and remains a critical cause of technique failure for some PD patients who must then be promptly switched to hemodialysis. Clinicians must identify risk factors associated with PD-related peritonitis. Peritonitis incidence has been significantly reduced in recent years owing to technological advances in PD connectology, development of new cycler machines and biocompatible PD solutions, and institution of the International Society for Peritoneal Dialysis (ISPD) guidelines for preventing and treating PD-related peritonitis ${ }^{8}$.

Previous reports have investigated the risk of peritonitis in patients on continuous ambulatory peritoneal dialysis (CAPD) versus automated peritoneal dialysis (APD) ${ }^{9-15}$. The available evidence is predominantly based on observational studies ${ }^{9-13}$ but not randomized control studies ${ }^{14,15}$ and is insufficient to evaluate the relationship between peritonitis risk and PD modality. The analysis of these studies is handicapped by failure to report on the connection device in the cyclers used. In addition, to our knowledge, there have been no investigations studying the relationship of CAPD and APD with peritonitis in Japan and with propensity score (PS) method. We therefore examined the association between PD modality at PD commencement and PD-related peritonitis through a multicentered cohort study in Japan with PS matching analysis.

\footnotetext{
${ }^{1}$ Department of Nephrology, Nara Prefecture General Medical Center, 2-897-5 Shichijo-nishimachi, Nara 630-8581, Japan. ${ }^{2}$ Department of Nephrology, Nara Prefecture Seiwa Medical Center, Nara 636-0802, Japan. ${ }^{3}$ Department of Nephrology, Nara Medical University, Kashihara, Nara 634-8521, Japan. ${ }^{凶}$ email: matsui-o-v2008420@ olive.plala.or.jp
} 


\begin{tabular}{|c|c|c|}
\hline $\begin{array}{c}\text { Nara } \\
\text { Medical } \\
\text { University } \\
(\mathrm{N}=242)\end{array}$ & $\begin{array}{c}\text { Nara } \\
\text { Prefecture } \\
\text { General Medical Center } \\
(\mathrm{N}=55)\end{array}$ & $\begin{array}{c}\text { Nara } \\
\text { Prefecture } \\
\text { Seiwa Medical Center } \\
(\mathrm{N}=40)\end{array}$ \\
\hline
\end{tabular}

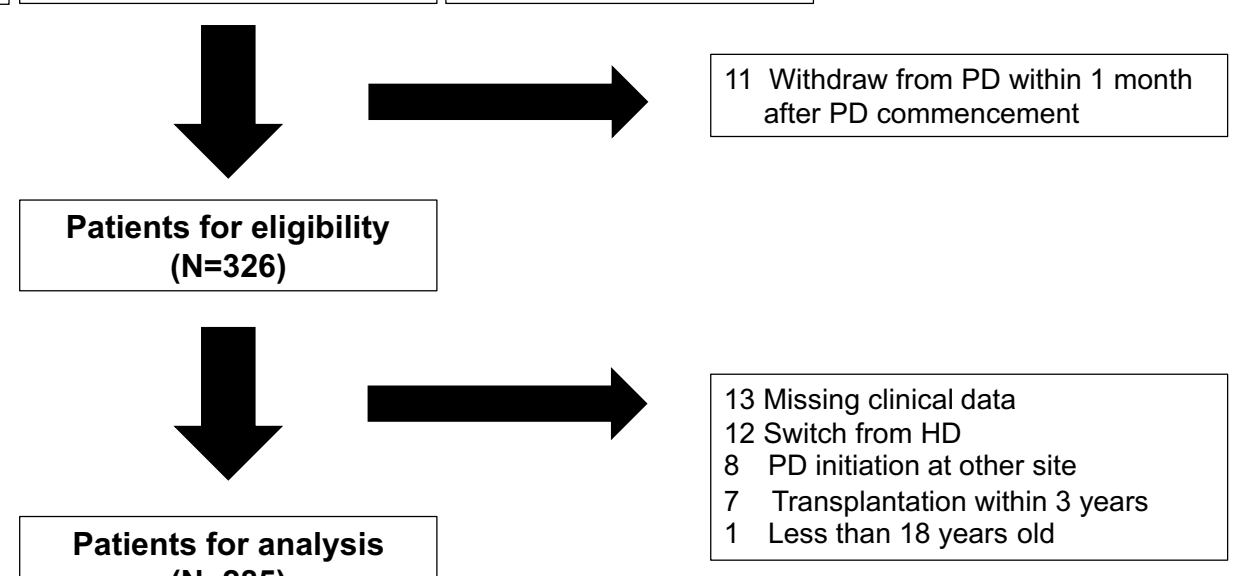

$(\mathrm{N}=\mathbf{2 8 5})$

Figure 1. Study Flowchart.

\begin{tabular}{|c|c|c|c|c|c|c|}
\hline & \multicolumn{2}{|c|}{ Before PS matching } & \multirow[b]{2}{*}{$p$ value } & \multicolumn{2}{|c|}{ After PS matching } & \multirow[b]{2}{*}{$p$ value } \\
\hline & APD & CAPD & & APD & CAPD & \\
\hline Number of patients (\%) & $133(47)$ & $152(53)$ & & $106(50)$ & $106(50)$ & \\
\hline Age, years & $63(54-72)$ & $62(53-73)$ & 0.85 & $61(53-72)$ & $63(53-73)$ & 0.93 \\
\hline Gender, female, n (\%) & $40(30)$ & $56(37)$ & 0.23 & $34(32)$ & $34(32)$ & 1.00 \\
\hline Diabetes, n (\%) & $67(50)$ & $52(34)$ & 0.006 & $46(43)$ & $43(41)$ & 0.68 \\
\hline Hypertension, n (\%) & $120(90)$ & $134(88)$ & 0.58 & $95(90)$ & $95(90)$ & 1.00 \\
\hline Dyslipidemia, n (\%) & $45(34)$ & $47(31)$ & 0.60 & $37(35)$ & $33(31)$ & 0.56 \\
\hline Overweight, n (\%) & $34(26)$ & $36(25)$ & 0.94 & $29(27)$ & $29(28)$ & 0.97 \\
\hline $\mathrm{eGFR}, \mathrm{mL} / \mathrm{min} / 1.73 \mathrm{~m}^{2}$ & $4.4(3.6-5.5)$ & $4.7(3.7-5.8)$ & 0.18 & $4.4(3.8-5.6)$ & $5.6(3.6-5.6)$ & 0.67 \\
\hline Hemoglobin, g/dL & $9.6(8.4-10.6)$ & $9.7(8.7-10.7)$ & 0.35 & $8.4(7.6-9.7)$ & $8.5(7.3-9.7)$ & 0.94 \\
\hline Serum albumin, $\mathrm{g} / \mathrm{dL}$ & $3.7(3.2-4)$ & $3.6(3.1-4)$ & 0.95 & $3.7(3.2-4.0)$ & $3.8(3.3-4.1)$ & 0.31 \\
\hline C-reactive protein, $\mathrm{mg} / \mathrm{dL}$ & $0.11(0.02-0.7)$ & $0.2(0.1-0.7)$ & 0.45 & $0.1(0-0.5)$ & $0.12(0.1-0.6)$ & 0.48 \\
\hline Uric acid, mg/dL & $7.8(6.4-9.3)$ & $7.8(6.3-9.4)$ & 0.76 & $8.1(6.3-9.3)$ & $7.9(6.3-9.5)$ & 0.90 \\
\hline Calcium, mg/dL & $8.7(7.7-9.2)$ & $8.8(8.3-9.3)$ & 0.03 & $8.8(7.8-9.2)$ & $8.6(8.0-9.1)$ & 0.88 \\
\hline Phosphorus, mg/dL & $5.8(5.0-7.3)$ & $5.7(4.6-6.7)$ & 0.07 & $5.8(4.9-6.9)$ & $6.0(4.8-6.9)$ & 0.91 \\
\hline Use of connection device, $\mathrm{n}(\%)(\%)$ & $77(58)$ & $69(45)$ & 0.03 & $59(56)$ & $55(52)$ & 0.58 \\
\hline
\end{tabular}

Table 1. Baseline characteristics before and after propensity score matching. Data are shown as the median (interquartile range) or $\mathrm{n}(\%)$ as appropriate. PS, propensity score; eGFR: estimated glomerular filtration rate.

\section{Results}

Baseline characteristics. Study flowchart was shown in Fig. 1. Two hundred eight-five PD patients (median age 62 years, interquartile range 60-63 years; 192 men) were analyzed in the present study. Table 1 lists their baseline characteristics at PD commencement. One hundred thirty-three patients were on APD; 152 were on CAPD. Diabetes prevalence and connecting device use were significantly higher in patients on APD than in those on CAPD. In our cohort of 205 patients with available solution data, use of $2.5 \%$ dextrose peritoneal dialysis solution bag was similar between APD (4\%) and CAPD ( $8 \%)$ patients $(p=0.25)$ but CAPD (27\%) patients have significantly high prevalence with use of icodextrin solution compared to APD $(6 \%)$ patients $(p<0.001)$.

The groups did not significantly differ after PS matching (Table 1).

Outcome and PD modality. During the study period (median 31 months), PD peritonitis occurred in 64 patients including 16 of 133 (0.05 episodes/patient-year) patients on APD and 48 of 152 (0.12 episodes/patientyear) patients on CAPD; using Poisson analysis this difference was significant $(p=0.005)$. In 56 patients with available causative organisms, the number and rate of gram-positive cocci showed a high prevalence in CAPD versus APD patients, but without statistical significance $(p=0.32)$ (Supplementary Figure). Among all patients, Kaplan-Meier analysis demonstrated that APD was significantly associated with a lower probability of peritoni- 
A

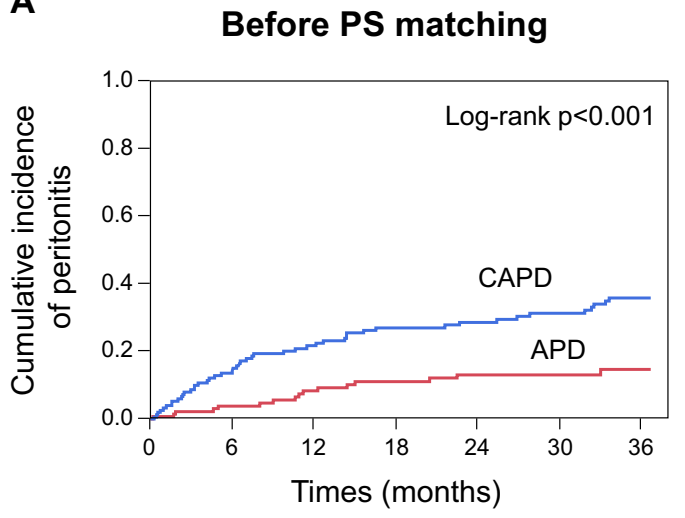

B

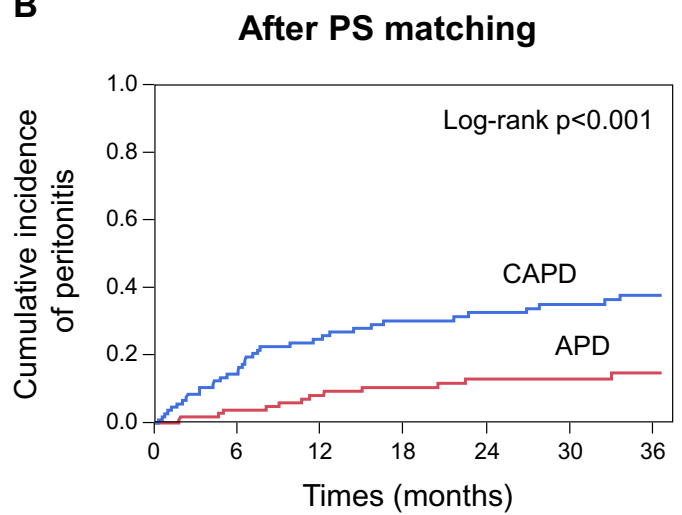

Figure 2. Kaplan-Meier analysis showing the cumulative incidence of the primary endpoint in patients receiving PD according to PD modality before $(\mathbf{A})$ and after $(\mathbf{B})$ propensity score matching $(p<0.001$ and $p<0.001$ by the log-rank test).

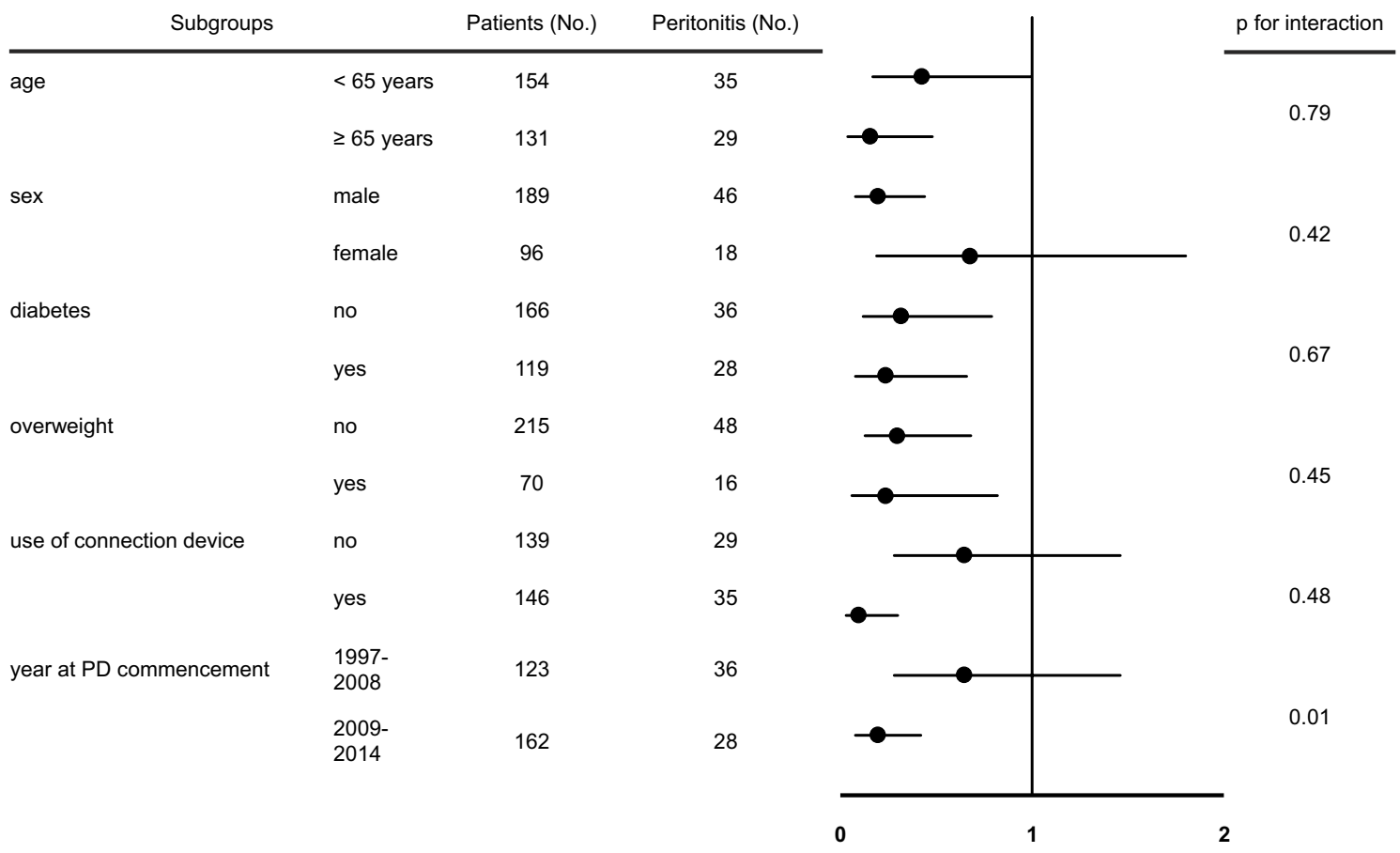

Figure 3. Multivariable-adjusted hazard ratios ( $95 \%$ confidence intervals) of the primary endpoints for APD versus CAPD within subgroups stratified by age, sex, diabetes, overweight patients and connection devise use.

tis than was CAPD (log-rank $p<0.001$; Fig. 2). Both the unadjusted and adjusted models showed that patients who started on APD had a lower risk of peritonitis. The hazard ratio (HR) and $95 \%$ confidence interval (CI) for the primary endpoint were $0.35(0.19-0.61)$ in the unadjusted model and was $0.33(0.19-0.59)$ in model 1 adjusted for age and sex, 0.32 (95\% CI 0.18-0.57) in model 2 adjusted for model 1 plus diabetes and overweight and 0.30 (95\% CI 0.17-0.53) in model 3 adjusted for model 2 plus use of connection device. The HR adjusted for the model 3 plus center remained significant (HR 0.30 [95\% CI 0.17-0.53]). In 205 participants with available solution data the HR was 0.28 (95\% CI 0.13-0.61) in the final model plus icodextrin solution.

After PS matching, APD continued to present a significantly lower risk of peritonitis than did CAPD (log-rank $p<0.001$, HR 0.32, 95\% CI 0.16-0.59; Fig. 1). We then used inverse probability of treatment weighting (IPTW) using PS to minimize the differences in patient characteristics. The IPTW-adjusted HR (95\% CI) for the primary outcome was 0.35 (0.18-0.67) for APD patients compared with CAPD patients.

The analyses stratified by age, sex, diabetes, overweight patients, connection device use and year at PD commencement, showed similar associations among participants, excluding female, no use of connection device and earlier years at PD commencement (Fig. 3). 


\section{Discussion}

Our study is the first to show that selecting APD rather than CAPD at PD commencement was significantly associated with a lower peritonitis incidence in Japanese PD patients. Adjusting for confounding covariates did not attenuate the HR of APD. Similar results were obtained in the PS-matching and IPTW analyses, suggesting that starting PD patients on APD rather than CAPD can reduce the risk of PD-associated peritonitis within 3 years after PD commencement.

Peritonitis is a major cause of PD failure via structural and functional alterations of the peritoneal membrane $\mathrm{e}^{16}$, which can lead to life-threatening events ${ }^{9,17}$. Previous reports have shown a relationship between PD modality and peritonitis, but these studies are observational ${ }^{9-13}$ rather than randomized control trials ${ }^{14,15}$. Results of many studies have shown that patients on APD have a lower or similar incidence of peritonitis than do those on CAPD. A randomized control study in the Netherlands revealed that PD peritonitis occurred significantly less often in APD versus CAPD patients ( 0.51 versus 0.94 episodes per patient-year, respectively $)^{14}$. A retrospective observational study in the United Kingdom ${ }^{9}$ showed that the peritonitis rate was 1:36.7 patient-months for APD-treated patients and 1:28.8 patient-months for CAPD-treated patients, representing an odds ratio of 0.78 favoring APD. Conversely, three recent observational studies found that PD modality was unassociated with a higher likelihood of developing peritonitis ${ }^{11-13}$. Differing study populations, follow-up times, improvements in CAPD and APD connection systems, and advances in nursing care and dialysis treatment may have led to the differing results in these studies. The analysis of these studies fails to report on the connection device in the cycler used. These data should also be interpreted with caution because of differences in the analytical methods. Our multicenter study in Japan showed that patients who started on APD at PD commencement experienced significantly less peritonitis than did those starting on CAPD in both the unmatched and matched patients and subgroup analyses, confirming the robustness of our results.

Two hypotheses may explain why APD was associated with a lower peritonitis incidence than was CAPD. First, the number of connections and disconnections required to perform PD may be the most important determinant of peritonitis rates; APD requires fewer connections and disconnections than does CAPD. Second, APD, especially nocturnal intermittent $\mathrm{PD}$, involves shorter dialysate dwelling times than does CAPD, which requires at least 4 to 5 bag exchanges. Fewer connections and shorter dwelling times may reduce the peritonitis incidence by reducing touch contamination in APD-treated patients. However, we found increased number and rate of peritonitis causing gram-positive cocci in CAPD versus APD patients, but without statistically significant. There may be other mechanisms that contribute to risk reduction of peritonitis in APD.

Several study limitations are noted. First, this was an observational study; therefore, the cause-effect relationship between APD and peritonitis incidence was uncertain. Second, the PD modality was selected by the clinicians and their patients, possibly introducing selection bias into the survey findings, although some clinical data were adjusted. Third, only the PD modality at PD commencement was analyzed; changes in PD modality were not followed. Fourth, the enrollment period was relatively long; thus, advances in dialysis treatment, including the dialysate and devices, might have affected the results. Based on results of the subgroup analysis, APD may contribute to risk reduction of peritonitis in recent years. Fifth, some variables associated with the choice of modality and peritonitis were not evaluated. Socioeconomic data, caregiver status and education level are associated with the choice of modality and incident peritonitis in PD patients ${ }^{18,19}$.

In conclusion, selection of APD rather than CAPD at PD commencement was associated with a lower peritonitis incidence, suggesting that APD may be preferable for prevention of peritonitis in PD patients. Larger prospective randomized studies are needed to ensure the robustness of our results.

\section{Methods}

Patients. In total, we screened 337 consecutive PD patients who were treated at three centers in Nara Prefecture in Japan between 1 April 1997 and 31 December 2014. Fifty-two patients were excluded because of the inclusion and exclusion criteria, leaving 285 patients for analysis. The inclusion criterion was that the patients must have received maintenance PD for at least one month. Exclusion criteria were missing clinical data, transition from hemodialysis, PD commencement at other clinics, transplantation within 3 years, and age less than 18 years. Each hospital's ethics board approved the study (Approval No. 2002-009, No. 316 and No.131).

Clinical definitions. Baseline demographics and blood sample results were obtained within one month before starting PD through patient interviews and medical records. Hypertension was defined as systolic blood pressure $\geq 140 \mathrm{mmHg}$, diastolic blood pressure $\geq 90 \mathrm{mmHg}$, or current treatment with oral antihypertensive drugs. Diabetes was defined as a fasting glucose level $\geq 126 \mathrm{mg} / \mathrm{dl}$ or current treatment with oral hypoglycemic medications or insulin. Dyslipidemia was defined as low-density lipoprotein cholesterol $\geq 140 \mathrm{mg} / \mathrm{dl}$ or current treatment with lipid-lowering medications.

Peritoneal dialysis. In the study PD system of two companies including Baxter Healthcare Corporation and Terumo Corporation was used; 253 (89\%) patients were on the system of Baxter Healthcare Corporation and the rest were on that of Terumo Corporation. Connection devices were UV Flash (Baxter Healthcare Corporation, Deerfield, Illinois, USA) and the TSCD (Terumo Sterile Connector Device, Terumo Corporation, Tokyo, Japan). The clinicians and patients determined whether to use CAPD or APD with or without a connection device.

Study outcomes. The primary outcome was time to first episode of peritonitis within 3 years after PD commencement. PD-related peritonitis was defined as an effluent leukocyte count of $>100 \mathrm{cells} / \mathrm{mm}^{3}$, with at least $50 \%$ being polymorphonuclear leukocytes according to ISPD guidelines ${ }^{8}$. In the entire cohort 2 patients 
with missing follow-up, 66 deaths and 81 patients who switched hemodialysis were treated as censored cases. All events were confirmed through medical records and self-reporting.

Statistical analysis. All variables are expressed as medians (interquartile range). Differences between the groups were determined using the Mann-Whitney $U$ test or the $\chi^{2}$ test. The rates of peritonitis between groups were compared by using Poisson analysis. Propensity scores were calculated using multivariable logistic regression to estimate probability of receiving APD versus CAPD. Demographics (age and sex), comorbidities (diabetes, hypertension, dyslipidemia and being overweight [defined as having a body mass index of $25 \mathrm{~kg} /$ $\mathrm{m}^{2}$ or more]), blood parameters (estimated glomerular filtration rate, hemoglobin, serum albumin, C-reactive protein, uric acid, calcium, and phosphorus), and use or no use of a connection device were included as covariates. Covariates were included in the propensity score model. Propensity scores were then used to match APD patients to CAPD patients 1:1 using a greedy nearest-neighbor matching algorithm. Cumulative incidence of the primary endpoint was estimated using the Kaplan-Meier method according to PD modality; differences were assessed using the log-rank test in both the unmatched and matched cohorts. A Cox regression model was used to determine unadjusted and adjusted associations between PD modality and the study endpoint. We initially adjusted for age and sex in model 1 . Model 2 consisted of model 1 plus diabetes and overweight and Model 3 consisted of model 2 plus use of connection device. Two-sided $p$ values $<0.05$ were considered statistically significant. JMP 10.0.02 (SAS Institute, Cary, NC, USA) and IBM SPSS Statistics, version 24 (IBM-SPSS Inc., Armonk, NY, USA) were used to perform all statistical analyses.

Ethical approval and informed consent. Nara Medical University, Nara Prefecture General Medical Center and Nara Prefecture Seiwa Medical Center Ethics Committee approved the study protocol and waived the requirement for written informed consent as a part of study approval (Approval No. 2002-009, No. 316 and No.131, respectively). All procedures performed in studies involving human participants were in accordance with the ethical standards of each institutional research committee and with 1964 Helsinki declaration and its amendments or comparable ethical standards. Research content has been included on the web page of our department (https://www.nara-hp.jp/about/ethics).

Received: 15 May 2020; Accepted: 2 September 2020

Published online: 30 October 2020

\section{References}

1. Fang, W. et al. Key factors for a high-quality peritoneal dialysis program-the role of the PD team and continuous quality improvement. Perit. Dial. Int. 34, S35-42. https://doi.org/10.3747/pdi.2013.00120 (2014).

2. Nishina, M. et al. A 10-year retrospective cohort study on the risk factors for peritoneal dialysis-related peritonitis: A single-center study at Tokai University Hospital. Clin. Exp. Nephrol. 18, 649-54. https://doi.org/10.1007/s10157-013-0872-y (2014).

3. Ghali, J. R. et al. Microbiology and outcomes of peritonitis in Australian peritoneal dialysis patients. Perit. Dial. Int. 31, 651-62. https://doi.org/10.3747/pdi.2010.00131 (2011).

4. Pérez Fontan, M. et al. Peritonitis-related mortality in patients undergoing chronic peritoneal dialysis. Perit. Dial. Int. 25, 274-84 (2005).

5. Fried, L. F. et al. Peritonitis influences mortality in peritoneal dialysis patients. J. Am. Soc. Nephrol. 7, 2176-2182 (1996).

6. Lin, C. Y. et al. Persistent transforming growth factor-beta 1 expression may predict peritoneal fibrosis in CAPD patients with frequent peritonitis occurrence. Am. J. Nephrol. 18, 513-519. https://doi.org/10.1159/000013397 (1998).

7. Margetts, P. J. et al. Inflammatory cytokines, angiogenesis, and fibrosis in the rat peritoneum. Am. J. Pathol. 160, 2285-2294. https ://doi.org/10.1016/S0002-9440(10)61176-5 (2002).

8. Li, P. K. I. S. P. D. et al. Update on prevention and treatment. Perit. Dial. Int. 36(481-508), 2016. https://doi.org/10.3747/ pdi.2016.00078 (2016).

9. Davenport, A. Peritonitis remains the major clinical complication of peritoneal dialysis: The London, UK, peritonitis audit 20022003. Perit. Dial. Int. 29, 297-302 (2009).

10. Balasubramanian, G. et al. Comparing automated peritoneal dialysis with continuous ambulatory peritoneal dialysis: Survival and quality of life differences?. Nephrol. Dial. Transpl. 26, 1702-1708. https://doi.org/10.1093/ndt/gfq607 (2011).

11. Lan, P. G. et al. The association between peritoneal dialysis modality and peritonitis. Clin. J. Am. Soc. Nephrol. 9, 1091-1097. https ://doi.org/10.2215/CJN.09730913 (2014).

12. Rüger, W. et al. Similar peritonitis outcome in CAPD and APD patients with dialysis modality continuation during peritonitis. Perit. Dial. Int. 31, 39-47. https://doi.org/10.3747/pdi.2009.00235 (2011).

13. Guilloteau, S. et al. Impact of assisted peritoneal dialysis modality on outcomes: A cohort study of the French language peritoneal dialysis registry. Am. J. Nephrol. 48(6), 425-433. https://doi.org/10.1159/000494664 (2018).

14. de Fijter, C. W. et al. Clinical efficacy and morbidity associated with continuous cyclic compared with continuous ambulatory peritoneal dialysis. Ann. Intern. Med. 120, 264-71. https://doi.org/10.7326/0003-4819-120-4-199402150-00002 (1994).

15. Bro, S. et al. A prospective, randomized multicenter study comparing APD and CAPD treatment. Perit. Dial. Int. 19, 526-533 (1999).

16. van Diepen, A. T. et al. The first peritonitis episode alters the natural course of peritoneal membrane characteristics in peritoneal dialysis patients. Perit. Dial. Int. 35, 324-32. https://doi.org/10.3747/pdi.2014.00277 (2015).

17. Boudville, N. et al. Recent peritonitis associates with mortality among patients treated with peritoneal dialysis. J. Am. Soc. Nephrol. 23, 1398-1405. https://doi.org/10.1681/ASN.2011121135 (2012).

18. Khalid, U. et al. "Educational" deprivation is associated with PD peritonitis. Perit. Dial. Int. 38, 251-256. https://doi.org/10.3747/ pdi.2017.00098 (2018).

19. Hsu, C. K. et al. Multidisciplinary predialysis education reduces incidence of peritonitis and subsequent death in peritoneal dialysis patients: 5-year cohort study. PLoS ONE 13, e0202781. https://doi.org/10.1371/journal.pone.0202781 (2018). 


\section{Acknowledgements}

We thank Akie Jimura, Hikaru Tasaki and Akiko Itano for contributing to the data collection. We thank E. Kanda for advising the statistical method. We thank Traci Raley, MS, ELS, from Edanz Group (www.edanzediting.com/ ac) for editing a draft of this manuscript.

\section{Author contributions}

$\mathrm{MK}, \mathrm{MM}$, and UT collected patients' data through medical records. MM contributed to conceiving and designing the study and analyzed and interpreted the data. KM, ME, KS, YA and KT contributed to writing and revising the manuscript. All authors reviewed the manuscript and approved the final version for publication.

\section{Competing interests}

This work was supported in part by Grants-in-Aid from the Ministry of Health, Labour and Welfare of Japan (No. 18K16005).

\section{Additional information}

Supplementary information is available for this paper at https://doi.org/10.1038/s41598-020-75918-5.

Correspondence and requests for materials should be addressed to M.M.

Reprints and permissions information is available at www.nature.com/reprints.

Publisher's note Springer Nature remains neutral with regard to jurisdictional claims in published maps and institutional affiliations.

Open Access This article is licensed under a Creative Commons Attribution 4.0 International License, which permits use, sharing, adaptation, distribution and reproduction in any medium or format, as long as you give appropriate credit to the original author(s) and the source, provide a link to the Creative Commons licence, and indicate if changes were made. The images or other third party material in this article are included in the article's Creative Commons licence, unless indicated otherwise in a credit line to the material. If material is not included in the article's Creative Commons licence and your intended use is not permitted by statutory regulation or exceeds the permitted use, you will need to obtain permission directly from the copyright holder. To view a copy of this licence, visit http://creativecommons.org/licenses/by/4.0/.

(C) The Author(s) 2020 